\title{
MODELAGEM DO PONTO DE ORVALHO E DO PERCENTUAL DE CONDENSAÇÃO PARA O GÁS DE RECICLO DE UM REATOR DE POLIMERIZAÇÃO DE ETENO EM LEITO FLUIDIZADO
}

\author{
K. P. N. PIRES ${ }^{1,2}$, J. B. V. BASTOS 2 , J. M. NIKIEL ${ }^{23}$ \\ ${ }^{1}$ Universidade Federal Fluminense, Faculdade de Engenharia Química \\ 2 Braskem S.A \\ ${ }^{3}$ Universidade Federal do Rio de Janeiro, Doutorando na Escola de Química
}

E-mail para contato: knossar@id.uff.br

\begin{abstract}
RESUMO - O presente trabalho apresenta uma ferramenta desenvolvida em uma unidade industrial da Braskem S.A para cálculo da temperatura do ponto de orvalho e do percentual de condensação dos componentes presentes no gás de fluidização de um reator de polimerização de eteno. Esta ferramenta é especialmente útil em momentos de indisponibilidade do módulo de controle avançado de processo da unidade (APC). O trabalho foi desenvolvido em três etapas: levantamento de dados; desenvolvimento de um cálculo iterativo utilizando a equação de Peng-Robinson para predição dos valores do ponto de orvalho da corrente gasosa; finalmente, para o calculo do percentual de condensação foi utilizado um ambiente de simulação para identificação da melhor função que descrevesse o comportamento da variável. Foram obtidos resultados satisfatórios para o percentual de condensação, após validação o modelo se tornou uma ferramenta útil para a garantia da continuidade operacional da unidade.
\end{abstract}

\section{INTRODUÇÃO}

Uma das tecnologias existentes para a produção do polietileno é a que utiliza um reator de leito fluidizado presente em um sistema de gás de reciclo onde as matérias-primas, em sua grande maioria, estão presentes em fase gasosa sendo representado pela Figura 1 retirada do trabalho de Alizadeh et al. (2003). Na corrente de entrada estão presentes alguns hidrocarbonetos de cadeias mais longas que o eteno, os comonômeros e o agente de condensação. Esses compostos, por serem menos voláteis, são alimentados a jusante do compressor o qual manterá a velocidade de fluidização do leito. Em seguida eles passam por um trocador de calor que é responsável pela remoção do calor de polimerização, e ali condensam. A parcela de gás condensada ao adentrar no reator vaporiza instantaneamente conferindo ao sistema uma maior capacidade de troca térmica e consequente aumento de produção. O modo de operação com condensação parcial do gás de reciclo é conhecido como Condensação Induzida. 
Figura 1 - Produção de polietileno em um reator de leito fluidizado

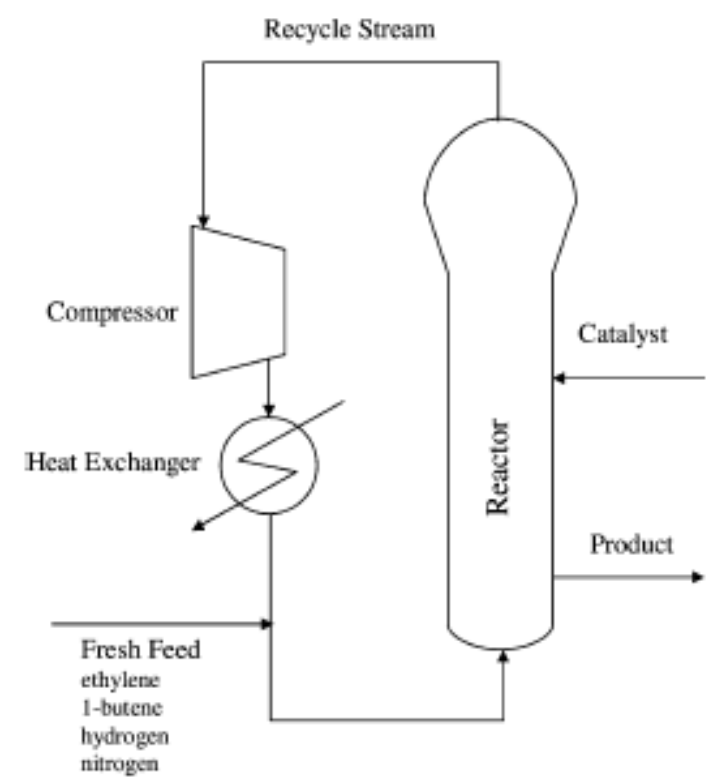

O Controle Avançado de Processo (APC) fornece uma série de informações indispensáveis à operação do reator. Dentre estas informações estão a temperatura do ponto de orvalho do gás de reciclo do reator e o percentual desse gás que condensa no trocador de calor e, portanto, auxilia a remoção de calor da reação. Desse modo, em momentos de crise, quando há uma falha na comunicação do sistema de produção com o APC, a planta passa a operar vulnerável a problemas de continuidade devido a falta de tais informações.

O controle do percentual de condensação do gás de reciclo é fundamental para o controle da carga do reator. Ao perder esta indicação a operação fica vulnerável a um aumento da condensação onde o excesso de líquido presente no gás pode se acumular sobre a placa distribuidora do reator que, ao se misturar com a resina polimérica, forma uma zona de lama. Essa lama formada leva a obstrução dos orifícios de passagem do gás, dificultando a fluidização do leito provocando a formação de aglomerados e podendo levar a uma parada inoportuna do equipamento e perdas significativas na produção. Já uma diminuição da quantidade de líquido no gás além de prejudicar a produção pode levar a problemas de continuidade por dificultar o controle da temperatura do reator.

\section{OBJETIVO}

Desenvolvimento de ferramenta para apoio operacional de uma unidade de polimerização de eteno da Braskem que utiliza a tecnologia de polimerização em leito fluidizado. 


\section{METODOLOGIA}

Para o desenvolvimento deste trabalho foi necessário o entendimento da forma que o APC realiza seus cálculos. O módulo utiliza a equação de estado BWR (Equação 2) que contém alto grau de complexidade devido às numerosas constantes utilizadas e que, em sua maioria, não eram fornecidas ou obtidas de forma direta, pois o módulo de controle é de propriedade do detentor da tecnologia e os cálculos não estão totalmente disponíveis ao usuário. Logo, optou-se por utilizar a equação de Peng-Robinson (Equação 1) por ser mais facilmente manuseada levando em conta os objetivos do trabalho.

O passo seguinte foi o levantamento de dados, tais como as propriedades dos gases presentes na corrente, quantidades e parâmetros de operação para ambientação do cenário crítico. Utilizou-se o software de simulação Aspen Plus ${ }^{\circledR}$ a fim de verificar o comportamento da mistura durante mudanças de temperatura.

Então, um modelo de cálculo iterativo, a partir da Equação 1, foi proposto para a obtenção da temperatura de orvalho. O modelo apresentou resultados satisfatórios quando comparado as informações produzidas pelo APC.

\section{RESULTADOS}

Para acompanhamento continuo da propriedade em questão por parte da equipe de operação da unidade foi criada uma tela no software de aquisição de dados do processo com interface simplificada a fim de facilitar a conferência da temperatura quando o Controle Avançado estiver fora de comunicação. A tela desenvolvida está demonstrada da Figura 2.

Figura 2- Tela para cálculo do ponto de orvalho

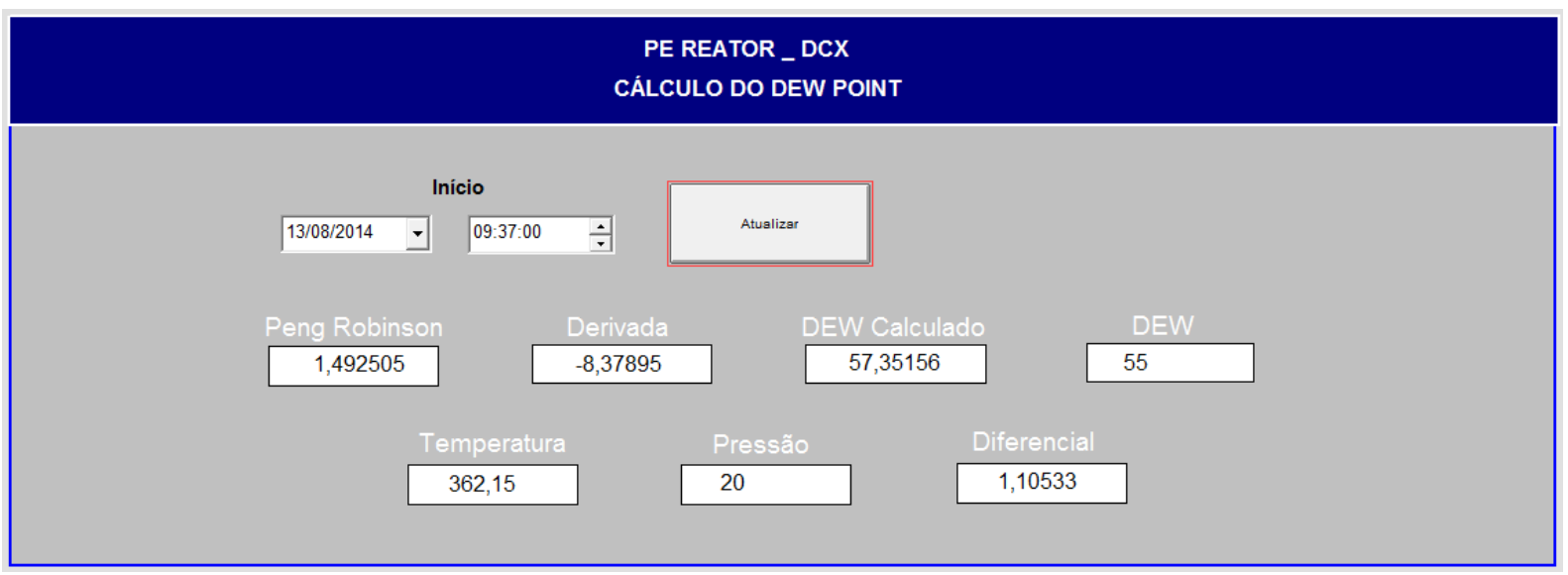

Com o propósito de mitigar ainda mais a vulnerabilidade do sistema um modelo para o cálculo do percentual de condensação foi desenvolvido.

A metodologia incialmente empregada no desenvolvimento do modelo do percentual de condensação foi semelhante ao descrito para determinação da temperatura de orvalho. Foram levantados os dados de todas as variáveis que poderiam influenciar no comportamento 
da variável em questão. Através de um software de simulação de processos os principais fatores foram relacionados, as respectivas constantes foram determinadas e, com isso, chegou-se em uma equação que descreveu a variável desejada com uma confiabilidade de $\underline{97 \%}$.

No Gráfico 1, é possível analisar o comportamento do modelo comparando-o com os dados reais coletados na planta. Observa-se que as curvas têm grande similaridade, o que vai de encontro com o objetivo do presente trabalho.

Gráfico 1 - Comparação dos valores do percentual de condensação

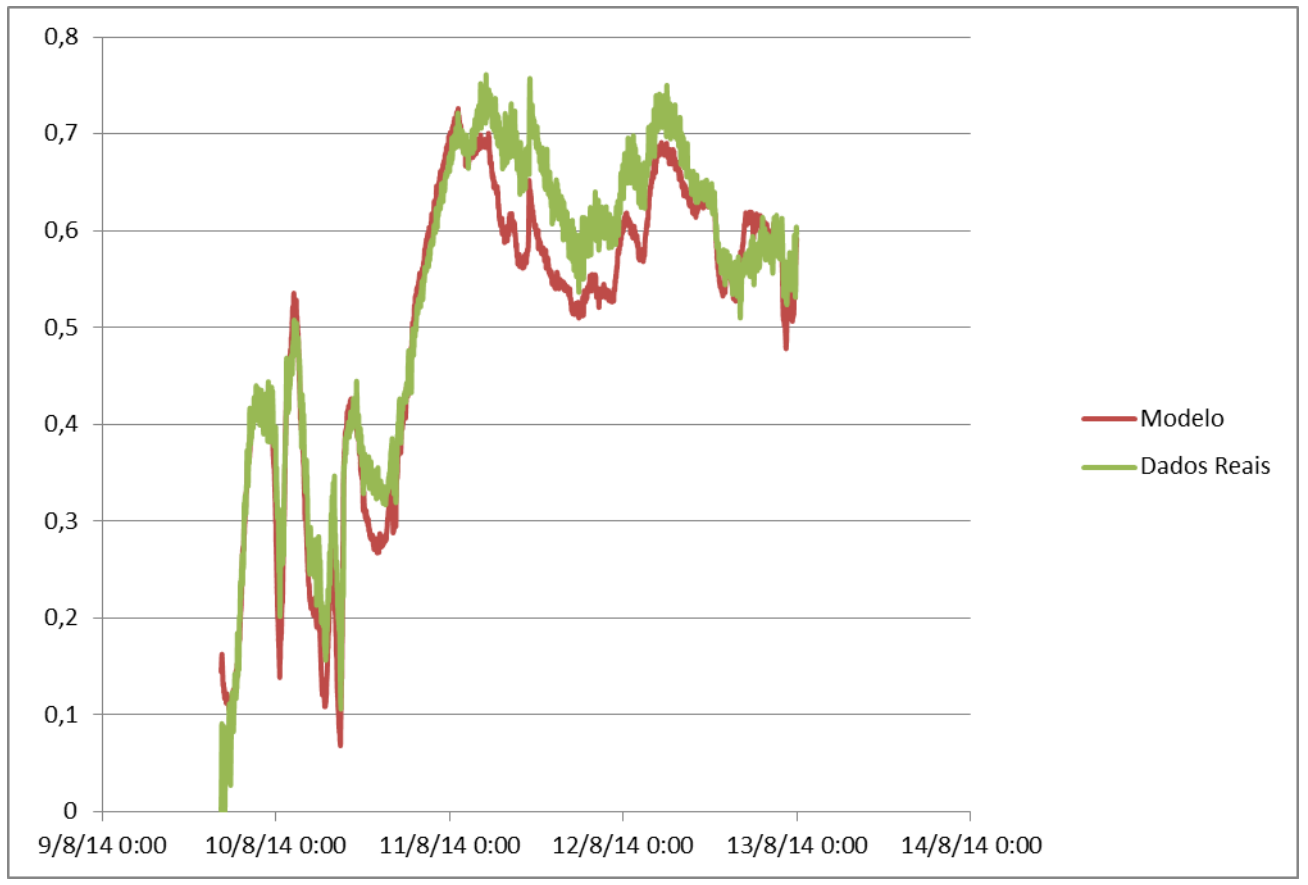




\section{EQUAÇÕES}

$$
\begin{aligned}
& P=\frac{R T}{V-b}-\frac{a(T)}{V^{2}+c b V+d b^{2}} \\
& p=\rho R T+\left(B_{0} R T-A_{0}-\frac{C_{0}}{T^{2}}+\frac{D_{0}}{T^{3}}-\frac{E_{0}}{T^{4}}\right) \rho^{2}+\left(b R T-a-\frac{d}{T}\right) \rho^{3}+\alpha\left(a+\frac{d}{T}\right) \rho^{6}+\frac{c \rho^{3}}{T^{2}}\left(1+\gamma \rho^{2}\right) \exp \left(-\gamma \rho^{2}\right)
\end{aligned}
$$

\section{NOMENCLATURA}
$\mathrm{a}, \mathrm{b}$
Constantes da Equação de Peng-Robinson
$\mathrm{B}_{0,}, \mathrm{~A}_{0}, \mathrm{C}_{\mathrm{o}}, \mathrm{a}, \mathrm{b}, \mathrm{c}, \alpha, \gamma$
Constantes da Equação de BWR
$\mathrm{P}$
Pressão absoluta $\left(\mathrm{kgf.cm}{ }^{-2} \cdot \mathrm{g}^{-1}\right)$
$\mathrm{R}$
Constante universal dos gases $\left(\mathrm{J} \cdot \mathrm{K}^{-1} \cdot \mathrm{mol}^{-1}\right)$
$\rho$
Densidade molar $\left(\mathrm{kg} \cdot \mathrm{mol}^{-1} \cdot \mathrm{m}^{-3}\right)$
$\mathrm{T}$
Temperatura absoluta (K)
V
Volume molar $\left(\mathrm{m}^{3} \cdot \mathrm{mol}^{-1}\right)$

\section{REFERÊNCIAS}

PERRY, H. R. Chemical Engineers's Handbook. $7^{\text {th }}$, Edition. McGrawn-Hill, 2000

SMITH, J. M. E VAN NESS, H. C. Introdução à Termodinâmica da Engenharia Química, terceira edição - Guanabara Dois, 1983.

ALIZADEH, M; MOUSTOFI, N A; PORMAHDIAN, S B; SOTUDEH-GHAREBAG, $\mathrm{R}$ a. Modeling of fluidized bed reactor of ethylene polymerization.

XIE, K.B. MCAULEY, J.C.C. HSU, D.W. BACON, Gas phase ethylene polymerization: preparation processes, polymer properties and reactor modelling, Ind. Eng. Chem. Res. 33 (1994) 449-479. 